This is an electronic reprint of the original article. This reprint may differ from the original in pagination and typographic detail.

Author(s): Idini, Andrea; Potel, G.; Barranco, F.; Vigezzi, E.; Broglia, R. A.

Title: Interweaving of elementary modes of excitation in superfluid nuclei through particlevibration coupling: Quantitative account of the variety of nuclear structure observables

Year: $\quad 2015$

Version:

Please cite the original version:

Idini, A., Potel, G., Barranco, F., Vigezzi, E., \& Broglia, R. A. (2015). Interweaving of elementary modes of excitation in superfluid nuclei through particle-vibration coupling: Quantitative account of the variety of nuclear structure observables. Physical Review C, 92(3), Article 031304.

https://doi.org/10.1103/PhysRevC.92.031304

All material supplied via JYX is protected by copyright and other intellectual property rights, and duplication or sale of all or part of any of the repository collections is not permitted, except that material may be duplicated by you for your research use or educational purposes in electronic or print form. You must obtain permission for any other use. Electronic or print copies may not be offered, whether for sale or otherwise to anyone who is not an authorised user. 


\title{
Interweaving of elementary modes of excitation in superfluid nuclei through particle-vibration coupling: Quantitative account of the variety of nuclear structure observables
}

\author{
A. Idini,,$^{1, *}$ G. Potel,${ }^{2,3, \dagger}$ F. Barranco, ${ }^{4, \dagger}$ E. Vigezzi, ${ }^{5,8}$ and R. A. Broglia ${ }^{6,7, \|}$ \\ ${ }^{1}$ Department of Physics, University of Jyvaskyla, FI-40014 Jyvaskyla, Finland \\ ${ }^{2}$ National Superconducting Cyclotron Laboratory, Michigan State University, East Lansing, MI 48824, USA \\ ${ }^{3}$ Lawrence Livermore National Laboratory, 7000 East Avenue, Livermore, California 94550, USA \\ ${ }^{4}$ Departamento de Física Aplicada III, Escuela Superior de Ingenieros, Universidad de Sevilla, Spain \\ ${ }^{5}$ INFN Sezione di Milano, I-20133 Milano, Italy \\ ${ }^{6}$ Dipartimento di Fisica, Università di Milano, I-20133 Milano, Italy \\ ${ }^{7}$ Niels Bohr Institute, University of Copenhagen, DK-2100 Copenhagen, Denmark \\ (Received 21 April 2015; revised manuscript received 28 July 2015; published 18 September 2015)
}

\begin{abstract}
A complete characterization of the structure of nuclei can be obtained by combining information arising from inelastic scattering, Coulomb excitation, and $\gamma$-decay, together with one- and two-particle transfer reactions. In this way it is possible to probe both the single-particle and collective components of the nuclear many-body wave function resulting from the coupling of these modes and, as a result, diagonalizing the low-energy Hamiltonian. We address the question of how accurately such a description can account for experimental observations in the case of superfluid nuclei. Our treatment goes beyond the traditional approach, in which these properties are calculated separately, and most often for systems near closed shells, based on perturbative approximations (weak coupling). It is concluded that renormalizing empirically and on equal footing bare single-particle and collective motion of open-shell nuclei in terms of self-energy (mass) and vertex corrections (screening), as well as particle-hole and pairing interactions through particle-vibration coupling (PVC), leads to a detailed, quantitative account of the data, constraining the possible values of the $k$ mass, of the ${ }^{1} S_{0}$ bare $N N$ interaction, and of the PVC strengths within a rather narrow window.
\end{abstract}

DOI: 10.1103/PhysRevC.92.031304

PACS number(s): 21.60.Jz, 23.40.-s, 26.30.-k, 21.30.Fe

Nuclear structure is both a mature [1-3] and a very active field of research [4,5], and the time seems ripe to attempt a balance of our present, quantitative understanding of it. Here we take up an aspect of this challenge and try to answer the question, how accurately can theory predict structure observables in terms of single-particle and collective degrees of freedom and of their couplings?

Each degree of freedom of a many-body system can be probed by a specific experiment. In the case of atomic nuclei, one-nucleon transfer processes give information on the singleparticle content of the levels under study, while two-particle transfer reactions probe the associated (particle-particle) pairing correlations. Finally, inelastic scattering, Coulomb excitation, and $\gamma$-decay probe (particle-hole) collective vibrations. Although this list looks like the contents of a review paper, the associated absolute differential cross sections and transition probabilities represent the minimum set of experimental information needed to characterize the structure of a nucleus, a direct consequence of PVC. Theory must account for all and each of them simultaneously in order to provide an accurate description of low-energy nuclear structure.

This is precisely what is accomplished in the present paper, in connection with an island of open-shell nuclei, namely,

\footnotetext{
*andrea.idini@gmail.com

†'gregory.potel@gmail.com

†barranco@us.es

§vigezzi@mi.infn.it

"broglia@mi.infn.it
}

$118,119,120,121,122 \mathrm{Sn}$, involved in the complete characterization of the spherical superfluid nucleus ${ }^{120} \mathrm{Sn}$. It is made operative by implementing the nuclear field theory (NFT) (see [6] and references therein) treatment of open-shell nuclei, based on the interplay of single-particle and collective vibrations. Making connections with other fields of physics, with all due provisos, here we carry out for superfluid nuclei, what is done in Chap. 7 of [7] for metals at low temperatures (field theoretical treatment of superconductivity), combined with Chap. 3 of the same reference (Cooper pair tunneling and Josephson effect). Summing up, the present paper differs from most works found in the nuclear structure literature, including those dealing with theories beyond mean field, in which usually specific nuclear structure aspects (e.g., single-particle motion) are considered, as a rule around closed shell systems (see [8] for a recent example).

It will be concluded that by making use of SLy4 effective interaction (mean field) and $v_{14}\left({ }^{1} S_{0}\right)$ Argonne pairing $N N$ potential, one is able to reproduce, within a $10 \%$ accuracy, a set of structure and reaction data which provides a complete characterization of ${ }^{120} \mathrm{Sn}$ and involves the island of open shell nuclei $118,119,120,121,122 \mathrm{Sn}$, with just three parameters. The strength $k_{2}$ and $k_{3}$ of the quadrupole and octupole multipole separable interactions [1] and a (relatively small) shift $\left(\left|\delta \epsilon_{d_{5 / 2}} /\left(\epsilon_{d_{5 / 2}}-\epsilon_{F}\right)\right| \approx 0.17\right)$ of the energy of the $d_{5 / 2}$ valence orbital. Within this context, the parameters $k_{2}$ and $k_{3}$ cannot be considered really free, being constrained by the energy and by the $\left(\beta_{\lambda}\right)_{0}[B(E \lambda)]$ experimental value of the lowest quadrupole and octupole collective vibrations of ${ }^{120} \mathrm{Sn}$.

The need for such a comprehensive approach, in particular in the case of open-shell nuclei, can be explained with the help 
of the following example. Pairing in nuclei has two sources. The ${ }^{1} S_{0}$ component of the strong (bare) $N N$ interaction (called $v_{p}^{\text {bare }}$ ) and the induced interaction $v_{p}^{\text {ind }}$. The latter results from the exchange of collective vibrations between time reversal states lying close to the Fermi energy (see $[9,10]$ and references therein), a mechanism similar to the electron-phonon coupling found in metallic superconductors [11-13]. It is certainly possible to adopt the simple BCS or Hartree-Fock-Bogoliubov (HFB) approximation with pure quasiparticle states, ignoring the coupling with vibrations (self-energy effects), as well as $v_{p}^{\text {ind }}$, and reproduce the experimental value of the pairing gap by properly adjusting the value of $v_{p}^{\text {bare }}$. On the other hand, the HFB approximation predicts at the same time that all of the ${ }^{120} \operatorname{Sn}(p, d){ }^{119} \operatorname{Sn}\left(5 / 2^{+}\right)$absolute cross section is concentrated in a single $d_{5 / 2}$ peak, and that only a single $5 / 2^{+} \rightarrow 3 / 2^{+} \gamma$-decay transition should be observed in the low-energy spectrum of ${ }^{119} \mathrm{Sn}$, in disagreement with the experimental findings. Concentrating on a single observable (pairing gap) or on a comprehensive set of observables (pairing gap, one-particle transfer absolute cross sections, $\gamma$-decay following Coulomb excitation) may lead to contradictory conclusions concerning the accuracy of a theoretical approach.

Let us now briefly dwell on some of the technical aspects of the present paper (for more details, see [14-17]). Single-quasiparticle and collective vibrations constitute the basis states. Consequently an embodiment of our theoretical description depends on the bare single-particle levels $\left(m_{k}\right)$, the bare pairing interaction $\left(v_{p}^{\text {bare }}\right)$, and the collectivity of the vibrational modes $\left(\beta_{\lambda}\right)$. In the present paper the calculations are implemented in terms of a SLy4 effective interaction [18] and a $v_{14}\left({ }^{1} S_{0}\right)\left(\equiv v_{p}^{\text {bare }}\right)$ Argonne pairing potential [19]. The first choice is guided by empirical evidence (elastic scattering analysis consistent with a $k$-mass of value $m_{k} \approx 0.7 \mathrm{~m}$ ). The second from the ${ }^{1} S_{0}$ phase shift analysis of $N N$ scattering. HFB provides the bare quasiparticle spectrum while QRPA a realization of density $\left(J^{\pi}=2^{+}, 3^{-}, 4^{+}, 5^{-}\right)$and $\operatorname{spin}\left(2^{ \pm}, 3^{ \pm}, 4^{ \pm}\right.$, $\left.5^{ \pm}\right)$modes. ${ }^{1}$ Taking into account renormalization processes (self-energy, vertex corrections, phonon renormalization, and phonon exchange) in terms of the PVC mechanism, the dressed particles as well as the induced pairing interaction $v_{p}^{\text {ind }}$ were calculated (see [14]; see also [20-27]). The Nambu-Gor'kov (NG) equation $[7,15,16,28,29]$ is then used to go beyond the weak-coupling approximation by propagating the different lowest-order NFT diagrams to infinite order, analogous to what is commonly done in the theory of metals [11-13] to take into account retardation and damping effects, which play a key role in a fundamental understanding of the superconducting phase [7]. Adding $v_{p}^{\text {ind }}$ to the bare interaction $v_{p}^{\text {bare }}$, the total pairing interaction $v_{p}^{\text {eff }}$ was determined. Solving the NG equation self-consistently using Green's function techniques the parameters characterizing the renormalized physical quasiparticle states are obtained.

The resulting values of the state-dependent pairing gap $\tilde{\Delta}_{v}=\tilde{\Delta}_{v}^{\text {bare }}+\tilde{\Delta}_{v}^{\text {ind }}$ are shown in Fig. 1(a). The contributions

\footnotetext{
${ }^{1}$ All the results obtained and discussed below remain essentially unchanged by considering as density modes only the $J^{\pi}=2^{+}$and $3^{-}$vibrations.
}

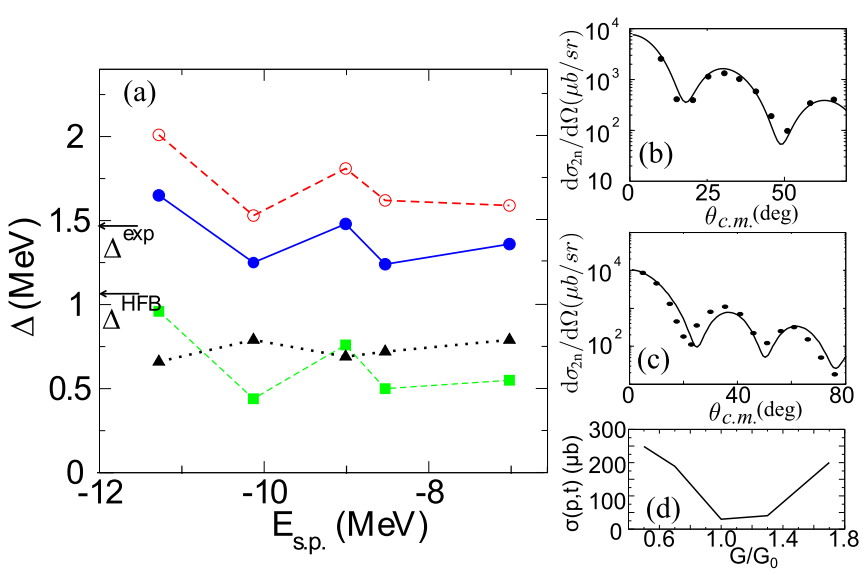

FIG. 1. (Color online) (a) State-dependent pairing gaps for the five valence orbitals of ${ }^{120} \mathrm{Sn}$. The value of $\Delta$ associated with the HFB solution of $v_{14}\left({ }^{1} S_{0}\right)\left(\equiv v_{p}^{\text {bare }}\right)$ is indicated by an arrow labeled $\Delta^{\mathrm{HFB}}$. The pairing gaps calculated making use of the empirically renormalized density modes are shown in terms of open circles joined by a dashed line, while the corresponding results obtained including also spin modes, and thus corresponding to $\tilde{\Delta}_{v}$ are shown by the solid dots joined by a continuous curve. The contributions $\tilde{\Delta}_{v}^{\text {bare }}$ and $\tilde{\Delta}_{v}^{\text {ind }}$ are displayed in terms of solid triangles and solid squares joined by dotted and by dashed lines respectively. (b),(c) Calculated two-particle transfer absolute differential cross sections associated with the reactions ${ }^{120} \mathrm{Sn}(p, t){ }^{118} \mathrm{Sn}$ (g.s.) and ${ }^{122} \mathrm{Sn}(p, t){ }^{120} \mathrm{Sn}$ (g.s.) (continuous curves) in comparison with experimental data (solid dots) $[38,39]$. (d) The absolute value of the deviation of the integrated theoretical absolute cross section from the experimental value in the case of the second reaction is given as a function of the strength of the bare pairing interaction (see footnote 3 below).

of $v_{p}^{\text {bare }}$ and $v_{p}^{\text {ind }}$ to $\tilde{\Delta}_{v}$ are about equal, density modes leading to attractive contributions which are partially canceled by spin modes, as expected from general transformation properties of the associated operators entering the particle-vibration coupling vertices [30-32]. Theory (SLY4+QRPA+(PVC) $\mathrm{REN}+\mathrm{NG}$ ) provides a quantitative account of the experimental value $\left(\Delta^{\exp } \approx 1.45 \mathrm{MeV}\right)$. It is to be noted that in carrying out the above calculations empirically renormalized collective modes have been adopted. ${ }^{2}$ This is because SLy4 leads to little collective density vibrations (see Table I, where, as an example, the bare QRPA results characterizing the low-lying $2^{+}$of ${ }^{120} \mathrm{Sn}$ are displayed [14]; see also [35]), in keeping with the associated value of the effective mass $0.7 \mathrm{~m}$. In fact, collectivity is closely associated with a density of levels $\left(\sim m^{*}\right)$

\footnotetext{
${ }^{2}$ These modes, in particular the low-lying quadrupole vibration used also in the calculation of the different quantities as a function of $\beta_{2}$, are determined as the QRPA solutions of a separable multipole-multipole interaction with empirical single-particle levels, adjusting the strength $k_{2}$ to obtain the desired properties [1]. Aside from being a simple shortcut for self-consistency in phonon renormalization, one avoids in this way the difficulties associated with the zero-range character (ultraviolet divergencies) and finite size instabilities of most Skyrme forces (see $[33,34]$ and references therein). This procedure is what is called empirical renormalization in the text.
} 
TABLE I. Energy, reduced E2 transition strength, and corresponding deformation parameter $\beta_{2}$ associated with the low-lying $2^{+}$state of ${ }^{120} \mathrm{Sn}$, calculated according to QRPA and empirically renormalized QRPA as explained in the text, and compared to experimental values [37]. The SLy4 single-particle levels were used in the calculation.

\begin{tabular}{lccc}
\hline \hline & $\hbar \omega_{2+}(\mathrm{MeV})$ & $B(E 2 \uparrow)\left(e^{2} \mathrm{fm}^{4}\right)$ & $\beta_{2}$ \\
\hline QRPA (SLy4) & 1.5 & 890 & 0.06 \\
QRPA + REN & 0.9 & 2150 & 0.14 \\
Expt. & 1.2 & 2030 & 0.13 \\
\hline \hline
\end{tabular}

consistent with an effective mass $m^{*}=m_{\omega} m_{k} / m \approx m$. This is achieved by coupling the two-quasiparticle (2qp) QRPA SLy4 solutions to 4qp doorway states made out of a $2 q p$ uncorrelated component and an empirically tuned (see footnote 2) QRPA collective mode (see Fig. 2 of [14]; see also [36]). The corresponding results are given in Table I, second and third lines; for details see [17].

To test the physical reliability of the results displayed in Fig. 1(a), we have recalculated $\tilde{\Delta}_{v}$ as a function of the quantities $\left\{m_{k}, v_{p}^{\text {bare }}, \beta_{2}\right\} .^{3,4}$ The results associated with the lowest quasiparticle state $h_{11 / 2}$, the leading orbital in determining the density of levels at the Fermi energy, are displayed in Figs. 2(a)-2(c). They provide evidence of the fact that a description based on the renormalization of single-particle states and collective modes through PVC leads to a global minimum for values of the set of quantities $\left\{m_{k}, v_{p}^{\text {bare }}, \beta_{2}\right\}$ equal to the empirical values: effective mass $m_{k} \approx 0.7 \mathrm{~m}$, bare pairing interaction strength $v_{p}^{\text {bare }}$ consistent with $v_{14}\left({ }^{1} S_{0}\right)$, and quadrupole deformation parameter $\left(\beta_{2}\right)_{0} \approx\left(\beta_{2}\right)_{\text {expt }} \approx 0.13$. The same conclusion can be reached concerning a variety of other observables. This can be seen from the results displayed in Figs. 1-4, as explained below. In Fig. 1(d), the deviation of the value of the calculated absolute two-nucleon transfer cross section $\sigma\left({ }^{122} \mathrm{Sn}(p, t){ }^{120} \mathrm{Sn}\right.$ (g.s.)) from the experimental value is shown as a function of the bare pairing interaction. In Fig. 2(d) the theoretical and experimental quasiparticle spectra associated with the ${ }^{120} \mathrm{Sn}$ valence orbitals $\left(h_{11 / 2}, d_{3 / 2}, s_{1 / 2}, g_{7 / 2}\right.$, and $\left.d_{5 / 2}\right)$ are shown as a function of $\beta_{2}$. In Figs. 2(e) and 2(f) the root mean-square deviation between

\footnotetext{
${ }^{3}$ In carrying out the calculations reported in Figs. 2(b) and 2(f), namely, the pairing gap $\Delta_{h 11 / 2}$ and the deviation of the quasiparticle spectrum from experiment as a function of the strength of the bare $N N$ pairing force $v_{14}\left({ }^{1} S_{0}\right)$ [19], the average value of the matrix elements of this interaction has been parametrized in terms of a monopole pairing force with constant matrix elements $G$. The reference value $G_{0}(\approx 0.22 \mathrm{MeV})$ has been determined so as to reproduce, in average, the $v_{14}\left({ }^{1} S_{0}\right)$ value of $\Delta_{j_{v}}$ of the valence states. In this way, one neglects the weak state dependence of the Argonne potential, of no consequence for the present discussion. The same protocol has been used to obtain the results displayed in Fig. 1(d).

${ }^{4}$ In the calculation of the pairing gap $\Delta_{h_{11 / 2}}$ as a function of $m_{k}$, the full NG solution was worked out for the Skyrme interactions Ska $\left(m_{k} / m=0.61\right)$, SAMi (0.67), SLy4 (0.70), SGII (0.79), SkM* (0.79), SkS1 (0.86), and SkP (1.0) and the corresponding quantity extracted [Fig. 2(a)].
}

theoretical and experimental quasiparticle energies is shown as a function of $\beta_{2}$ and of the ratio $G / G_{0}$ respectively. In Fig. 2(g) the splitting of the multiplet of states $\left(h_{11 / 2} \otimes 2^{+}\right)_{15 / 2^{-}-7 / 2^{-}}$ averaged over the ${ }^{119} \mathrm{Sn}$ and ${ }^{121} \mathrm{Sn}$ spectrum is shown as a function of $\beta_{2}$, while the resulting root mean-square deviation with respect to the experimental value is displayed in Fig. 2(h). In Fig. 3(a) the experimental $\gamma$-decay spectrum following Coulomb excitation of ${ }^{119} \mathrm{Sn}$ [37] is compared with the theoretical one [calculated with $\beta_{2}=\left(\beta_{2}\right)_{0}$, Fig. 3(b)], while the root mean-square deviation between experiment and theory is shown in Fig. 3(c) as a function of $\beta_{2}$. Finally, in Fig. 4(d) the centroid and width of the $5 / 2^{+}$strength function obtained from one-particle transfer are shown as a function of $\beta_{2}$.

The comprehensiveness of the above findings is likely to be one of the most far reaching achievements of the present paper. Concerning single, specific points, we can mention that (a) the quasiparticle spectrum shown in Fig. 2(d) compares favorably with a recent shell model study of the energetics of the $\mathrm{Sn}$ isotopes and associated electromagnetic transition probabilities [41]; (b) the splitting of the $h_{11 / 2} \otimes 2^{+}$[Fig. 2(g)] compares also at profit with results of early work solely dedicated to this subject [42] and leading to a somewhat too large splitting; (c) our calculations of the $d_{5 / 2}$ strength function [Fig. 4(c)] reproduce experiment more accurately than done in previous attempts (see [43] and references therein); and (d) the calculations which are at the basis of the results shown in Fig. 3 display similarity with those of Ref. [44], solely dedicated to a $\gamma$-decay study of ${ }^{117} \mathrm{Sn}$ and carried out making use of the quasiparticle phonon model.

Because of the PVC mechanism, the different valence quasiparticle states undergo renormalization and fragmentation, phenomena which can be specifically probed with one-particle transfer reactions. In Fig. 4(a) we display the absolute differential cross sections associated with the reaction ${ }^{120} \mathrm{Sn}(d, p){ }^{121} \mathrm{Sn}(l j)$, calculated making use of the spectroscopic amplitudes associated with the strongest populated fragments of the valence orbitals $h_{11 / 2}, d_{3 / 2}, s_{1 / 2}$, and $d_{5 / 2}$ and of global optical parameters [45], in comparison with the experimental data [46]. Theory provides a quantitative account of the experimental findings. It is of notice that the agreement found between the summed absolute differential cross sections associated with the almost degenerate state $3 / 2^{+}$and $11 / 2^{-}$(experimentally nonresolvable [46], while theoretically separated by $100 \mathrm{keV}$ ), results from a subtle incoherent combination of the $l=2, d \sigma_{1 n} / d \Omega$ peaked at $\theta_{\text {c.m. }} \approx 20^{\circ}$ and of that of the $l=5$ one at $\theta_{\text {c.m. }}=47^{\circ}$.

In discussing the ${ }^{120} \mathrm{Sn}(p, d){ }^{119} \mathrm{Sn}$ reaction [47] we concentrate on the $d_{5 / 2}$ orbital, the most theoretically challenging of all of the valence single-particle strength functions. This is because this state, being farther away from the Fermi energy $\left(\epsilon_{d_{5 / 2}}=-11.3 \mathrm{MeV}, \epsilon_{F} \approx-8 \mathrm{MeV}\right)$ than the other four valence orbitals $\left(\epsilon_{g_{7 / 2}}=-10.1, \epsilon_{s_{1 / 2}}=-9.0, \epsilon_{d_{3 / 2}}=\right.$ -8.5 , and $\left.\epsilon_{h_{11 / 2}}=-7.1 \mathrm{MeV}\right)$, is embedded in a denser set of doorway states (of type $s_{1 / 2} \otimes 2^{+}, d_{3 / 2} \otimes 2^{+}, g_{7 / 2} \otimes$ $2^{+}, h_{11 / 2} \otimes 3^{-}$, etc.). Consequently, it can undergo accidental degeneracy and thus conspicuous fragmentation. As seen from Table II, although the calculated summed cross sections $(\sigma=6.15 \mathrm{mb})$ agree, within experimental errors, with observation $(7.93 \pm 2 \mathrm{mb})$, theory predicts an essentially uniform 

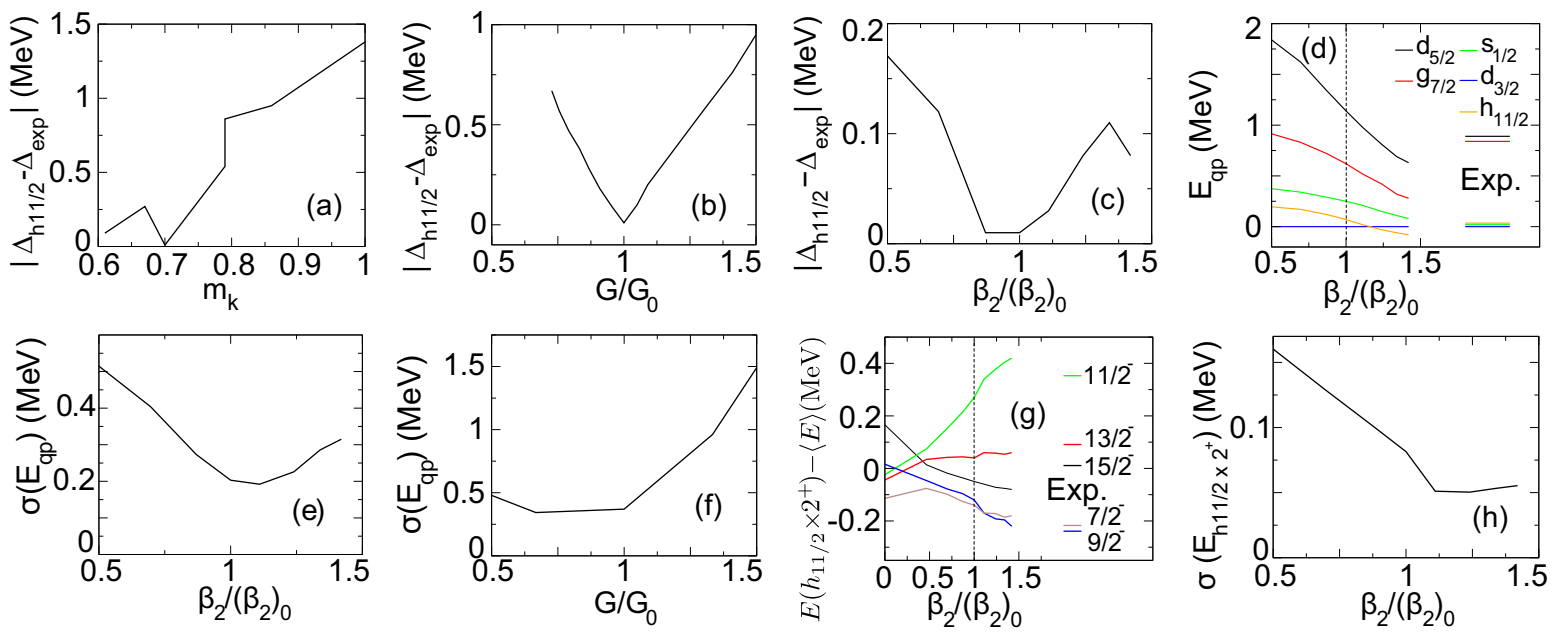

FIG. 2. (Color online) Absolute value of the difference between the experimental pairing gap and the theoretical value of $\Delta_{h_{11 / 2}}$ calculated as a function of (a) the effective mass $m_{k}$ associated with different Skyrme forces (see footnote 4), (b) the ratio $G / G_{0}$ (see footnote 3), and (c) the ratio $\beta_{2} /\left(\beta_{2}\right)_{0}$ (see footnote 2). (d) The lowest quasiparticle energy values as a function of $\beta_{2} /\left(\beta_{2}\right)_{0}$ obtained from the full calculation as explained in the text referred to the energy of the $3 / 2^{+}$state, in comparison with the experimental data. (e) Root mean-square deviation between the experimental and theoretical levels shown in (d) as a function of $\beta_{2} /\left(\beta_{2}\right)_{0}$. (f) Root mean-square deviation between the experimental and theoretical energies, shown in (d) as a function of the ratio $G / G_{0}$. (g) The experimental energies of the members of the $h_{11 / 2} \otimes 2^{+}$multiplet compared with the theoretical values, calculated as a function of the ratio $\beta_{2} /\left(\beta_{2}\right)_{0}$. (h) Root mean-square deviation between the experimental and theoretical energies of the members of the $h_{11 / 2} \otimes 2^{+}$multiplet shown in (g) as a function of $\beta_{2} /\left(\beta_{2}\right)_{0}$.

fragmentation of the strength over an energy interval of $\approx 760$ $\mathrm{keV}$, while the data [47] are consistent with a concentration of the strength at an energy close to that of the lowest theoretical $5 / 2^{+}$level.

In keeping with the above scenario we have shifted the bare SLy4 single-particle energy $\epsilon_{d_{5 / 2}}$ by $600 \mathrm{keV}[(-11.3+$ 0.6) $\mathrm{MeV}=-10.7 \mathrm{MeV}]$, amounting to a modest overall change in the bare density of valence levels. Making use of the corresponding nuclear structure results and of global optical parameters [45], we have then recalculated all the quantities discussed above, in particular the absolute differential cross sections associated with the $5 / 2^{+}$states lying below $2 \mathrm{MeV}$ and populated in the reaction ${ }^{120} \mathrm{Sn}(p, d){ }^{119} \mathrm{Sn}$.

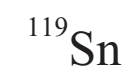

(a)

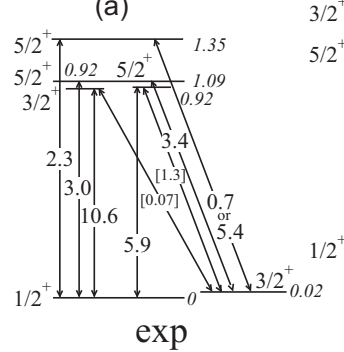

FIG. 3. (a) Experimental $B(E 2)$ values [37] in units of $B_{\text {sp }}$, of the quadrupole $\gamma$-decay following ${ }^{119} \mathrm{Sn}\left(\alpha, \alpha^{\prime}\right){ }^{119} \mathrm{Sn}^{*}$ Coulomb excitation, connecting the low-lying states of ${ }^{119} \mathrm{Sn}$. (b) Corresponding theoretical values calculated making use of the results of the full renormalized calculation as explained in the text. The energies are in $\mathrm{MeV}$. (c) Root mean-square deviation between the experimental transition strengths associated with $E 2$ decay from the $5 / 2^{+}$levels, and the theoretical values calculated as a function of the $\beta_{2}$ parameter.
They are displayed in Fig. 4(b) (left panel) in comparison with the experimental data (right panel). Theory now provides a quantitative account of the experimental findings. In particular, of the fact that the strength function is dominated by a single peak. With the $600 \mathrm{keV}$ shift, it is predicted at an energy of $1050 \mathrm{keV}$ carrying $4.4 \mathrm{mb}$ and it is observed at $1090 \mathrm{keV}$ with a cross section of $5.35 \pm 1.3 \mathrm{mb}$ (Table II). The resulting overall agreement between theory and experiment is further confirmed by Fig. 4(c) where the value of the absolute one-particle transfer strength function associated with the population of $5 / 2^{+}$states predicted by the calculation is compared with experiment.

Making again use of the effective occupation numbers resulting from the solution of the NG equation, the two-nucleon spectroscopic amplitudes of the reactions ${ }^{120} \mathrm{Sn}(p, t){ }^{118} \mathrm{Sn}$ (g.s.) and ${ }^{122} \mathrm{Sn}(p, t){ }^{120} \mathrm{Sn}$ (g.s.) have been worked out. With the help of these quantities and of global optical parameters [45], the value of the absolute differential cross sections have been calculated in second-order DWBA taking into account successive and simultaneous transfer, properly corrected from nonorthogonality contributions [45]. They are displayed in Figs. 1(b) and 1(c) in comparison with the experimental findings $[38,39]$. Theory reproduces the value of the absolute cross sections associated with the ground state transitions of two-particle transfer reactions within experimental errors (integrated cross section of ${ }^{120} \mathrm{Sn}(p, t){ }^{118} \mathrm{Sn}: 2250 \pm$ $338 \mu \mathrm{b}$ (theory: $2190 \mu \mathrm{b}$ ); ${ }^{122} \mathrm{Sn}(p, t){ }^{120} \mathrm{Sn}: 2505 \pm 376 \mu \mathrm{b}$ (theory: $2466 \mu b$ ) ) [45]. In the case in which one uses the HFB approximation with pure (SLy4) quasiparticle states adjusting the value of $v_{p}^{\text {bare }}$ to reproduce the value of the pairing gap one obtains 1880 and $2055 \mu \mathrm{b}$, respectively.

The calculations have been repeated for different values of the strength of the PVC associated with the most important 


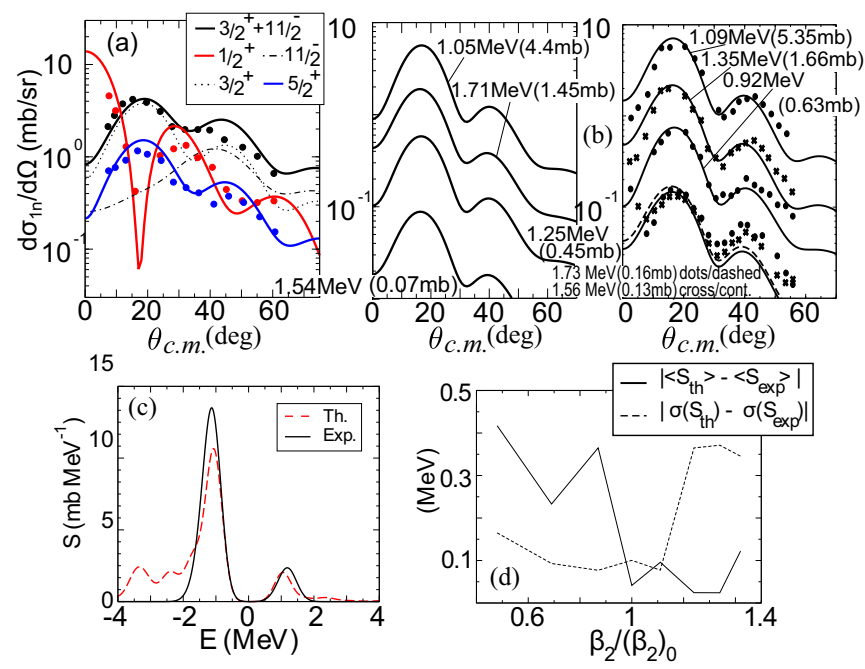

FIG. 4. (Color online) (a) Absolute finite range, full recoil DWBA theoretical differential cross sections associated with the low-lying fragments of the $h_{11 / 2}, d_{3 / 2}, s_{1 / 2}$, and $d_{5 / 2}$ valence states most strongly populated in the reaction ${ }^{120} \mathrm{Sn}(d, p){ }^{121} \mathrm{Sn}$, calculated with the help of state-of-the-art optical potentials and $v_{n p}$ interaction [40], using the NG structure input, in comparison with the experimental data [46]. In the NG calculations the $d_{5 / 2}$ single-particle orbit in the SLy 4 mean-field potential has been shifted toward $\epsilon_{F}$ by $0.6 \mathrm{MeV}$ (see text). (b) ${ }^{120} \mathrm{Sn}(p, d){ }^{119} \mathrm{Sn}\left(5 / 2^{+}\right)$absolute experimental differential cross sections [47], together with the DWBA fit used in the analysis of the data (right panel) in comparison with the DWBA calculations (left panel) carried out as mentioned in (a). (c) Comparison of the calculated strength function $S_{5 / 2}\left\{d\left[\sigma\left({ }^{120} \operatorname{Sn}(p, d){ }^{119} \operatorname{Sn}\left(5 / 2^{+}\right)\right)+\right.\right.$ $\left.\left.\sigma\left({ }^{120} \operatorname{Sn}(d, p)^{121} \operatorname{Sn}\left(5 / 2^{+}\right)\right)\right] / d E\right\}$ with experimental data $[46,47]$. The peaks have been folded with a Gaussian function of variance $0.25 \mathrm{MeV}$. (d) The difference between the centroid (width) of the experimental and of the calculated $d_{5 / 2}$ strength $S_{5 / 2}$ is shown as a function of the ratio $\beta_{2} /\left(\beta_{2}\right)_{0}$ in terms of the solid (dashed) curve.

TABLE II. Most prominent experimental (theoretical) fragments of the $d_{5 / 2}$ single-particle state populated in the ${ }^{120} \mathrm{Sn}(p, d){ }^{119} \mathrm{Sn}$ $\left(5 / 2^{+}\right)$reaction lying below $2 \mathrm{MeV}$, calculated using the $\mathrm{NG}$ equation and the bare SLy4 single-particle levels in comparison with the data [47]. The energies (in $\mathrm{keV}$ ) are listed in the first (fourth) column, while the absolute cross sections (in $\mathrm{mb}$ ) are given in the second (fifth), integrated within the range $2^{\circ}<\theta_{\text {c.m. }}<55^{\circ}$, and third (sixth), peak cross section (in mb/sr), $\left(\theta_{\text {c.m. }}\right)_{\max } \approx 17^{0}$. In the last three columns, similar results are displayed but now calculated using the shifted energy value $\left(\epsilon_{d_{5 / 2}}=-10.7 \mathrm{MeV}\right)$ of the $d_{5 / 2}$ valence orbital.

\begin{tabular}{|c|c|c|c|c|c|c|c|c|}
\hline \multirow[t]{2}{*}{$\epsilon_{i}$} & \multicolumn{2}{|c|}{ Expt. } & \multicolumn{3}{|c|}{ SLy4 } & \multicolumn{3}{|c|}{ SLy4 $\left(d_{5 / 2}\right.$ shift $)$} \\
\hline & $\sigma$ & $d \sigma / d \Omega$ & $\epsilon_{i}$ & $\sigma$ & $d \sigma / d \Omega$ & $\epsilon_{i}$ & $\sigma$ & $d \sigma / d \Omega$ \\
\hline 921 & 0.63 & 0.75 & & & & & & \\
\hline 1090 & 5.35 & 7.0 & 1150 & 1.80 & 2.3 & 1050 & 4.40 & 5.60 \\
\hline 1354 & 1.66 & 2.3 & 1290 & 1.20 & 1.7 & 1250 & 0.45 & 0.58 \\
\hline 1562 & 0.13 & 0.16 & 1710 & 0.25 & 0.32 & 1540 & 0.07 & 0.09 \\
\hline \multirow[t]{2}{*}{1730} & 0.16 & 0.18 & 1910 & 2.90 & 4.0 & 1710 & 1.45 & 1.90 \\
\hline & $7.93 \pm 2$ & 10.39 & & 6.15 & 8.32 & & 6.37 & 8.17 \\
\hline
\end{tabular}

TABLE III. Root mean-square deviation $\sigma$ between the experimental data and the theoretical values taken at the minimum of the corresponding functions displayed in Figs. 1(d), 2(c), 2(e), 2(h), 3(c), and 4(d) reported in $\mathrm{keV}$ for the pairing gap, quasiparticle energies, multiplet splitting, centroid, and width of the $5 / 2^{+}$low-lying singleparticle strength distribution. In single-particle units $B_{\mathrm{sp}}$ for the $\gamma$-decay $\left[B(E 2)\right.$ transition probabilities] and in $\mu \mathrm{b}$ for $\sigma_{2 n}(p, t)$. In brackets the ratio $\sigma / L$, called $\sigma_{\text {rel }}$ in the text, between $\sigma$ and the experimental range $L$ of the corresponding quantities $[1.4 \mathrm{MeV}(\Delta)$, $1 \mathrm{MeV}\left(E_{\mathrm{qp}}\right), 800 \mathrm{keV}$ (mult. splitting), $1 \mathrm{MeV}\left(d_{5 / 2}\right.$ centroid), $809 \mathrm{keV}$ (=1730-921) $\mathrm{keV}$ ( $d_{5 / 2}$ width), $10 B_{\mathrm{sp}}(B(E 2)), 2505 \mu \mathrm{b}$ $\left.\left(\sigma_{2 n}(p, t)\right)\right]$, is given.

\begin{tabular}{lcc}
\hline \hline Observables & SLy4 & $d_{5 / 2}$ shift \\
\hline$\Delta$ & $10(0.7 \%)$ & $10(0.7 \%)$ \\
$E_{\mathrm{qp}}$ & $190(19 \%)$ & $160(16 \%)$ \\
Mult. splitt. & $50(7 \%)$ & $70(10 \%)$ \\
$d_{5 / 2}$ strength (centr.) & $200(20 \%)$ & $40(4 \%)$ \\
$d_{5 / 2}$ strength (width) & $160(20 \%)$ & $75(9.3 \%)$ \\
$B(E 2)$ & $1.4(14 \%)$ & $1.34(13 \%)$ \\
$\sigma_{2 n}(p, t)$ & $40(2 \%)$ & $40(2 \%)$ \\
\hline \hline
\end{tabular}

collective vibrational mode, namely, the lowest $2^{+}$as well as for different strengths of the bare pairing interaction. While the dependence of $\sigma_{2 n}(p, t)$ is very weak with $\beta_{2}$ (not shown), it is conspicuous with $v_{p}^{\text {bare }}$. An example of such dependence is displayed in Fig. 1(d).

Within this context, it is of notice that a measure of the reliability with which theory can describe the nuclear structure is provided by the relative dimensionless standard deviations $\sigma_{\text {rel }}$ associated with each of the different observables, as shown in Table III.

We conclude that a theoretical description of nuclear structure based on single-particle (mean field with $m_{k} \approx 0.7 \mathrm{~m}$ ), on the associated collective motion (QRPA), and on their interweaving controlled by the particle-vibration coupling mechanism which leads to renormalization of both types of nuclear excitations through mass (self-energy) and screening (vertex) corrections as well as to induced pairing to be added to $v_{14}\left({ }^{1} S_{0}\right)$ can provide a quantitative account, within a $10 \%$ average error level (see Table III), of the nuclear structure representative of a mass zone (group of nuclei displaying homogeneous properties such as sphericity and superfluidity, likely circumscribed by phase transition domains). The PVC mechanism is found to play a central role in achieving this result. The above considerations and protocols are not only transferable to the remaining superfluid Sn isotopes (not considered explicitly in the present case), but also applicable to the quantitative description of other spherical, superfluid nuclear mass zones.

Suggestions and insight provided by P. F. Bortignon are gratefully acknowledged. This work has been supported by the Academy of Finland and University of Jyvaskyla within the FIDIPRO program and by the Helmholtz Association through the Nuclear Astrophysics Virtual Institute (VH-VI417) and the Helmholtz International Center for FAIR within the framework of the LOEWE program launched by the State of Hesse. 
[1] A. Bohr and B. R. Mottelson, Nuclear Structure, Vol. II (Benjamin, New York, 1975).

[2] A. Bohr, Rotational Motion in Nuclei, Le Prix Nobel en 1975, Norstedts Tryckeri, Stockholm (1976) 56.

[3] B. R. Mottelson, Elementary Modes of Excitation in $\mathrm{Nu}$ clei, Le Prix Nobel en 1975, Norstedts Tryckeri, Stockholm (1976) 80.

[4] Proceedings of the International Nuclear Physics Conference, Florence 2013, edited by S. Lunardi et al., EPJ Web Conf. 66 (2014).

[5] 50 Years of Nuclear BCS, edited by R. A. Broglia and V. Zelevinsky (World Scientific, Singapore, 2013).

[6] P. F. Bortignon, R. A. Broglia, D. R. Bès, and R. Liotta, Nuclear field theory, Phys. Rep. 30, 305 (1977).

[7] J. Schrieffer, Superconductivity (Benjamin, New York, 1964).

[8] D. Tarpanov, J. Dobaczewski, J. Toivanen, and B. G. Carlsson, Spectroscopic Properties of Nuclear Skyrme Energy Density Functional, Phys. Rev. Lett. 113, 252501 (2014).

[9] F. Barranco, R. A. Broglia, G. Gori, E. Vigezzi, P. F. Bortignon, and J. Terasaki, Surface Vibrations and Pairing Interactions in Nuclei, Phys. Rev. Lett. 83, 2147 (1999).

[10] D. Brink and R. A. Broglia, Nuclear Superfluidity (Cambridge University Press, Cambridge, UK, 2005).

[11] J. Bardeen and D. Pines, Electron-phonon interaction in metals, Phys. Rev. 99, 1140 (1955).

[12] A. Migdal, Interaction between electrons and lattice vibrations in a normal metal, Sov. Phys. JETP 7, 996 (1958).

[13] H. Fröhlich, Theory of the superconducting state. I. The ground state at the absolute zero of temperature, Phys. Rev. 79, 845 (1950).

[14] F. Barranco, R. A. Broglia, G. Colò, G. Gori, E. Vigezzi, and P. F. Bortignon, Many-body effects in nuclear structure, Eur. Phys. J. A 21, 57 (2004).

[15] A. Idini, F. Barranco, and E. Vigezzi, Quasiparticle renormalization and pairing correlations in spherical superfluid nuclei, Phys. Rev. C 85, 014331 (2012).

[16] A. Idini, Renormalization effects in nuclei, Ph.D. thesis, University of Milan, 2013, http://air.unimi.it/2434/216315.

[17] F. Barranco et al. (unpublished).

[18] E. Chabanat et al., A Skyrme parametrization from subnuclear to neutron star densities, Nucl. Phys. A 627, 710 (1997); A Skyrme parametrization from subnuclear to neutron star densities Part II. Nuclei far from stabilities, 635, 231 (1998).

[19] R. B. Wiringa, R. A. Smith, and T. L. Ainsworth, Nucleonnucleon potentials with and without $\Delta$ (1232) degrees of freedom, Phys. Rev. C 29, 1207 (1984).

[20] A. V. Avdeenkov and S. P. Kamerdzhiev, Description of excitations in odd nonmagic nuclei by Green's function method, Phys. At. Nucl. 62, 563 (1999).

[21] S. P. Kamerdzhiev, A. V. Avdeenkov, and D. A. Voitenkov, Quasiparticle-phonon interaction in the theory of finite Fermi systems, Phys At. Nucl. 74, 1478 (2011).

[22] E. V. Litvinova and A. V. Afanasjev, Dynamics of nuclear single-particle structure in covariant theory of particle-vibration coupling: From light to superheavy nuclei, Phys. Rev. C 84, 014305 (2011).

[23] E. Litvinova, Quasiparticle-vibration coupling in a relativistic framework: Shell structure of $Z=120$ isotopes, Phys. Rev. C 85, 021303 (2012).
[24] P. Ring and E. Litvinova, Particle vibrational coupling in covariant density functional theory, Phys. At. Nucl. 72, 1285 (2009).

[25] G. Colò, H. Sagawa, and P. F. Bortignon, Effect of particlevibration coupling on single-particle states: A consistent study within the Skyrme framework, Phys. Rev. C 82, 064307 (2010).

[26] K. Mizuyama, G. Coló, and E. Vigezzi, Continuum particlevibration coupling method in coordinate-space representation for finite nuclei, Phys. Rev. C 86, 034318 (2012).

[27] N. V. Gnezdilov, I. N. Borzov, E. E. Saperstein, and S. V. Tolokonnikov, Self-consistent description of single-particle levels of magic nuclei, Phys. Rev. C 89, 034304 (2014).

[28] D. Van Neck, M. Waroquier, V. Van der Sluys, and J. Ryckebusch, Proton hole states in ${ }^{208} \mathrm{~Pb}$, studied by means of a self-consistent solution of the second-order Dyson equation for single-particle propagators, Nucl. Phys. A 563, 1 (1993).

[29] V. Somà, C. Barbieri, and T. Duguet, Ab initio self-consistent Gorkov-Green's function calculations of semi-magic nuclei: Numerical implementation at second order with a two-nucleon interaction, Phys. Rev. C 89, 024323 (2014).

[30] P. F. Bortignon, R. A. Broglia, and C. H. Dasso, Quenching of the mass operator associated with collective states in many-body systems, Nucl. Phys. A 398, 221 (1983).

[31] H.-J. Schulze, J. Cugnon, A. Lejeune, M. Baldo, and U. Lombardo, Medium polarization effects on neutron matter superfluidity, Phys. Lett. B 375, 1 (1996).

[32] H. Heiselberg, C. J. Pethick, H. Smith, and L. Viverit, Influence of Induced Interactions on the Superfluid Transition in Dilute Fermi Gases, Phys. Rev. Lett. 85, 2418 (2000).

[33] V. Hellemans et al., Spurious finite-size instabilities in nuclear energy density functionals, Phys. Rev. C 88, 064323 (2013).

[34] A. Pastore, D. Tarpanov, D. Davesne, and J. Navarro, Spurious finite-size instabilities in nuclear energy density functionals: Spin channel, arXiv:1505.05043.

[35] J. Terasaki, J. Engel, and G. F. Bertsch, Systematics of the first $2^{+}$excitation in spherical nuclei with the Skryme quasiparticle random-phase approximation, Phys. Rev. C 78, 044311 (2008).

[36] G. Bertsch, P. F. Bortignon, and R. A. Broglia, Damping of nuclear excitations, Rev. Mod. Phys. 55, 287 (1983).

[37] P. H. Stelson, W. T. Milner, F. K. McGowan, R. L. Robinson, and S. Raman, Coulomb excitation of ${ }^{117,119}$ Sn, Nucl. Phys. A 190, 197 (1972).

[38] P. Guazzoni, L. Zetta, A. Covello, A. Gargano, B. F. Bayman, T. Faestermann, G. Graw, R. Hertenberger, H.-F. Wirth, and M. Jaskola, ${ }^{118} \mathrm{Sn}$ levels studied by the ${ }^{120} \mathrm{Sn}(p, t)$ reaction: Highresolution measurements, shell model, and distorted-wave Born approximation calculations, Phys. Rev. C 78, 064608 (2008).

[39] P. Guazzoni et al., Level structure of ${ }^{120} \mathrm{Sn}$ : High resolution $(p, t)$ reaction and shell model description Phys. Rev. C 60, 054603 (1999).

[40] I. J. Thompson (private communication).

[41] T. Engeland, M. Hjorth-Jensen, M. Kartamyshev, and E. Osnes, The Kuo-Brown effective interaction: from ${ }^{18} \mathrm{O}$ to the $\mathrm{Sn}$ isotopes, Nucl. Phys. A 928, 51 (2014).

[42] A. Kuriyiama, T. Marumori, and K. Matsuyanagi, Structure of the anomalous coupling states with spin $I=(j-1)$, Prog. Theor. Phys. Suppl. 58, 53 (1975).

[43] D. G. Fleming, A $20 \mathrm{MeV}(d, p)$ study of nuclear structure in the even and odd tin isotopes, Can. J. Phys. 60, 428 (1982). 
[44] J. Bryssink et al., Search for the electric dipole excitations to the $3 s_{1 / 2} \otimes\left[2_{1}^{+} \otimes 3_{1}^{-}\right]$multiplet in ${ }^{117} \mathrm{Sn}$, Phys. Rev. C 62, 014309 (2000).

[45] G. Potel, A. Idini, F. Barranco, E. Vigezzi, and R. A. Broglia, Cooper pair transfer in nuclei, Rep. Prog. Phys. 76, 106301 (2013).
[46] M. J. Bechara and O. Dietzch, States in ${ }^{121} \mathrm{Sn}$ from the ${ }^{120} \mathrm{Sn}(d, p){ }^{121} \mathrm{Sn}$ reaction at $17 \mathrm{MeV}$, Phys. Rev. C 12, 90 (1975).

[47] S. Dickey, J. Kraushaar, R. Ristinen, and M. Rumore, The ${ }^{120} \mathrm{Sn}$ $(p, d){ }^{119} \mathrm{Sn}$ reaction at $26.3 \mathrm{MeV}$, Nucl. Phys. A 377, 137 (1982). 